\title{
Intracoronary Imaging in the Management of a Complex and Recurrent Acute Coronary Syndrome Associated With Multiple Comorbidities. A Case Report
}

\author{
Ioana Rodean, Elisabeta Himcinschi, Alexandra Tirca, Daniel Cernica \\ Department of Computational Imaging, Cardio Med Medical Center, Tîrgu Mureș, Romania
}

\section{ABSTRACT}

Coronary artery disease represents a major cause of morbidity and mortality around the world. Unstable angina pectoris is a serious manifestation of ischemic heart disease and represents an acute condition caused by the narrowing of the coronary lumen as the result of an atheromatous plaque formation. In most cases the trigger of this process is represented by the rupture of a plaque that has become vulnerable or unstable. The first-line intracoronary imaging technique for the evaluation of plaque vulnerability is optical coherence tomography, which can measure the thickness of the fibrous cap (a significant predictor of plaque vulnerability) and can also assess other characteristics of plaque vulnerability (macrophage infiltration, lipid pool, intracoronary thrombus, or neointimal rupture). We present the case of a 67-year-old male with symptoms suggestive of unstable angina pectoris, caused by the presence of a vulnerable plaque on the left main coronary artery, where optical coherence tomography had a significant contribution in identifying the etiology of chest pain.

Keywords: acute coronary syndrome, vulnerable plaque, optical coherence tomography, coronary stenosis, angiography

\section{ARTICLE HISTORY}

Received: May 10, 2017

Accepted: June 5, 2017

\section{CORRESPONDENCE}

Elisabeta Himcinschi

Str. 22 Decembrie 1989 nr. 76

540124 Tîrgu Mureș, Romania

Tel: +40 265217333

E-mail: eli_himcinschi@yahoo.com

\section{INTRODUCTION}

Coronary artery disease (CAD) represents a major cause of morbidity and mortality around the world, contributing to the death of an estimated $13 \%$ of the global population. ${ }^{1,2}$ Unstable angina pectoris (UA) is a serious manifestation of ischemic heart disease and represents an acute condition produced by the narrowing of the coronary lumen as the result of an atheromatous plaque formation. The majority of UA cases are triggered by the rupture of a previously stable coronary plaque that has become vulnerable or unstable. Coronary plaques have often been studied in relation to their location, severity, and functional impact. At present, all modifications in the structure of a plaque can be analyzed using newly developed imaging techniques. ${ }^{3,4}$ Over the past decades, several major advances in the diagnosis and treatment of ischemic heart disease have been encountered; nevertheless, invasive coronary angiography remains the gold standard for diagnosing a coronary 
artery stenosis. Since its outset more than 40 years ago, several adjunctive techniques have been initiated in order to optimize the overall diagnostic accuracy and also to prevent the inter- and intra-observer variability related to the estimation of lesion severity. A novel invasive technology dedicated either to evaluate the physiological significance or the anatomical and morphological features of coronary lesions, optical coherence tomography (OCT), enriches the arsenal of diagnostic tools available to an interventional cardiologist. As an alternative to these invasive methods, noninvasive imaging techniques, such as multi-detector-row computed tomography (MDCT) or magnetic resonance imaging (MRI), have been developed, currently serving as the most promising noninvasive imaging tests for the diagnosis of coronary artery disease. ${ }^{5}$

In this paper, we present the case of a patient with multiple associated comorbidities, admitted to the cardiology clinic several times for multiple recurrent acute episodes of ischemic coronary artery disease, where modern imaging technologies allowed the establishment of a complex diagnosis and the initiation of appropriate therapeutic procedures.

\section{CASE REPORT}

A 67-year-old male, with a history of multivessel coronary artery disease associated with type 2 non-insulindependent diabetes mellitus and stage 3 chronic kidney disease, was admitted to the Cardio Med Medical Center in
Tîrgu Mureș, Romania, presenting constrictive chest pain with sudden onset, lasting more than 20 minutes, radiating in the left upper limb, and associated with rest dyspnea and one episode of Adam-Stokes syncope.

Seven years prior to this presentation, the patient underwent a coronary angiography, which revealed an acute thrombotic stenosis on the anterior descending coronary artery (Figure 1A), treated successfully by the implantation of a drug-eluting stent with optimal results and TIMI III post-procedural flow (Figure 1B).

The patient gave informed consent allowing the publication of his data, and the institution where the patient had been admitted approved the publication of the case.

In addition to the typical signs and symptoms of acute coronary syndrome (ACS) mentioned above, physical examination revealed stage 2 arterial hypertension (blood pressure value 160/100 $\mathrm{mmHg}$ ) and chronic heart failure.

Laboratory investigations showed elevated levels of serum biomarkers characterizing hepatic, renal, and pancreatic function such as: ALAT: $56 \mathrm{IU} / \mathrm{L}$, ASAT: $123 \mathrm{IU} / \mathrm{L}$, eGFR: $48.8-54.6 \mathrm{~mL} / \mathrm{min} / 1.73 \mathrm{~m} 2$ (Cockroft-Gault formula), blood glucose: $220 \mathrm{mg} / \mathrm{dL}$. The electrocardiogram showed sinus rhythm, intermediate QRS axis, a heart frequency of 64 beats/minute, and the presence of a major right bundle branch block.

Coronary angiography revealed a minimal in-stent restenosis in the previously implanted stent and the development of new lesions in all three major coronary arteries: a $70 \%$ stenosis in the distal part of the left main coronary

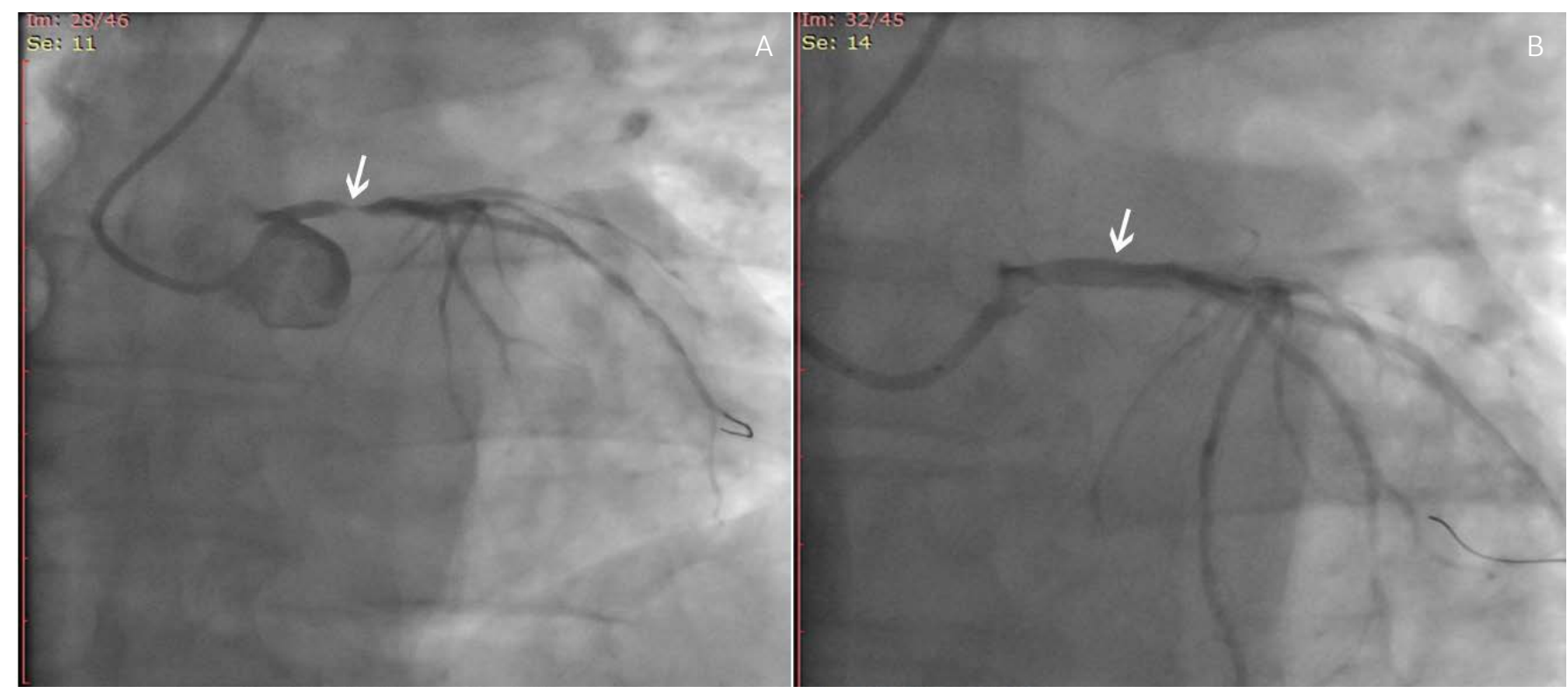

FIGURE 1. Coronary angiography. A - significant stenosis in the left coronary artery (arrow); B - post-intervention aspect, with no residual stenosis (arrow) 


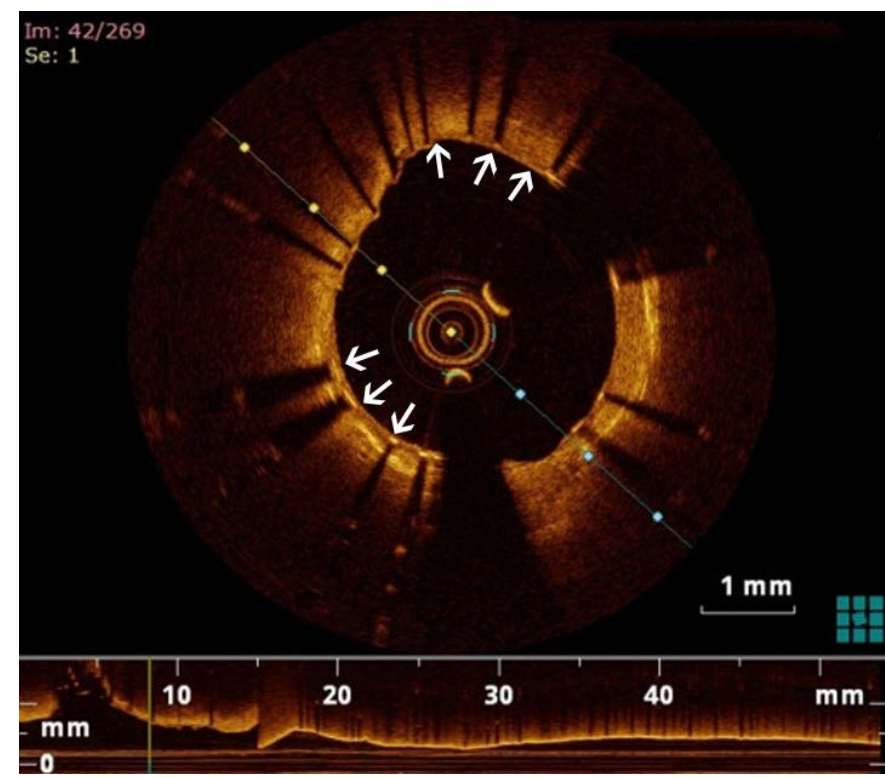

FIGURE 2. Optical coherence imaging showing a coronary stent well apposed to the arterial wall, with no signs of in-stent restenosis (arrows)

artery (Figure $1 \mathrm{~A}$ ), a 50\% stenosis on the left anterior descending coronary (LAD), an ostial stenosis of the intermediary branch and a 70\% long stenosis of the proximal right coronary artery.

The patient received interventional treatment consisting in the implantation of a $3.5 \times 12 \mathrm{~mm}$ drug-eluting stent in the left main coronary artery (Figure 1B), a 2.75 $\times 15 \mathrm{~mm}$ drug-eluting stent in the intermediary branch, and a $3 \times 23 \mathrm{~mm}$ drug-eluting stent in the right coronary artery, with optimal results and TIMI III flow in all territories. The lesion in the LAD remained untreated as it was not hemodynamically significant.

Following the interventional treatment, the patient was treated with anti-ischemic drugs, lipid-lowering drugs, diuretics, and antiarrhythmic drugs, with a favorable evolution.

At the 1-year follow-up, the patient reported the onset of a new chest pain for which he underwent an MRI study, an echocardiographic assessment, and a new coronary angiography. Despite several myocardial ischemic events, MRI showed no evidence of myocardial fibrosis of the left ventricle, and all structural and functional parameters of the myocardium were within normal range. Echocardiography revealed a calcified posterior mitral ring, a stage I/ II mitral regurgitation, a stage I aortic regurgitation, lowered contractility of the left ventricle, decreased motility of the lateral wall of the left ventricle, and increased echogenicity of the posterior part of the pericardium. Coronary angiography identified new lesions in the coronary tree, however not significant, namely a 50\% stenosis in the third segment of the anterior descending coronary artery and a minimal in-stent restenosis, without any indication for revascularization.

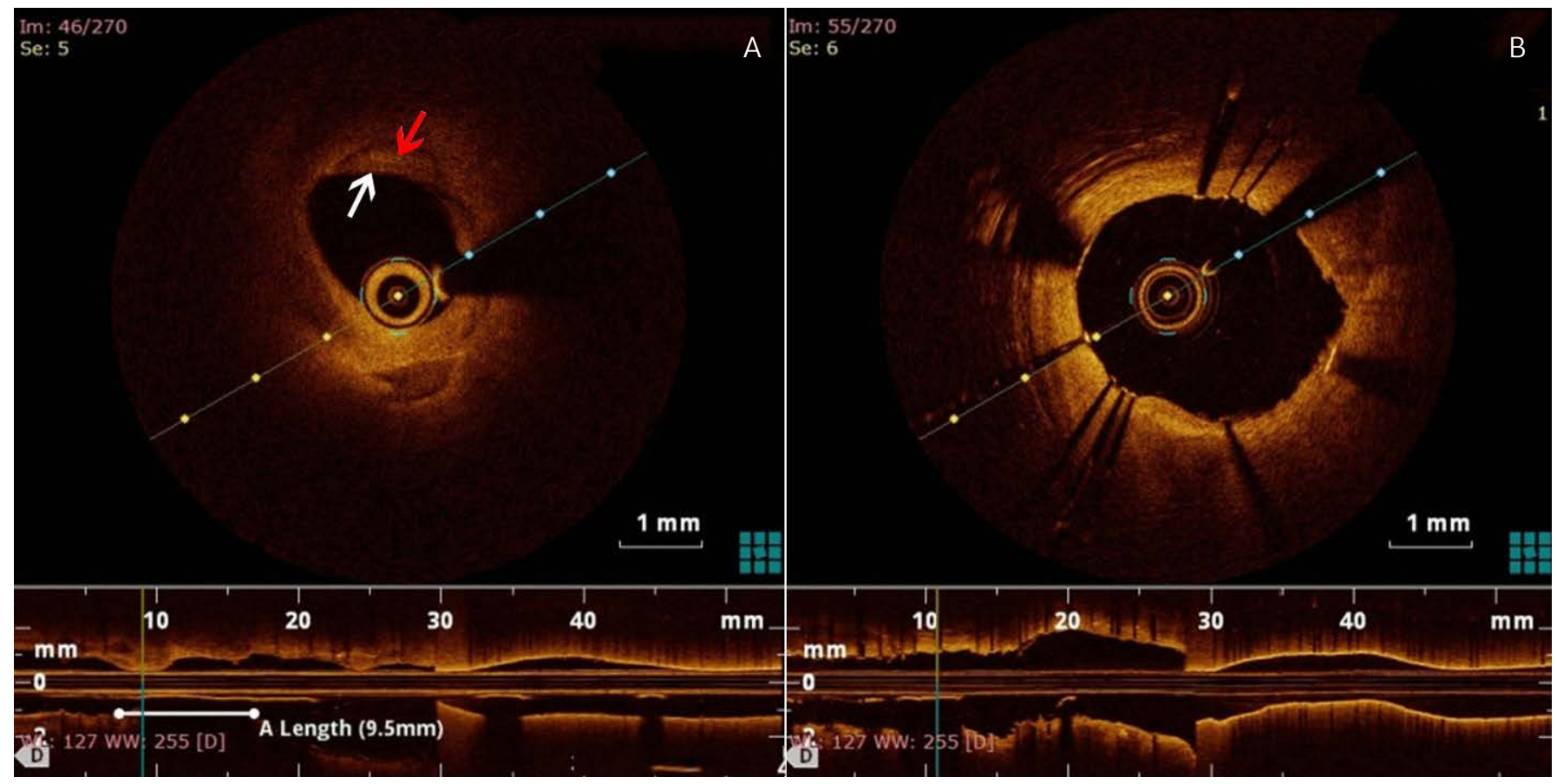

FIGURE 3. Optical coherence tomography distal to the stent implanted in the LAD. A - significant vulnerable coronary plaque (arrow); B - plaque treated by implantation of a coronary stent (arrow), well apposed to the vessel wall and with no plaque protrusion 
After 3 weeks, the patient returned with acute chest pain, therefore a new coronary angiography was performed in emergency, which did not reveal any significant difference compared to the previous examination from an angiographic point of view. Therefore, an OCT examination was performed in order to evaluate in detail all new coronary lesions and to detect a possible vulnerable plaque responsible for the symptomatology. OCT showed a left main stent with well-apposed struts and good intimal coverage, without neointimal hyperplasia or neoatherosclerotic plaque inside the stented segment (Figure 2). At the same time, OCT visualized an atherosclerotic plaque in the proximal LAD, characterized by a large lipid pool and a thin fibrous cap of 18 microns, being therefore classified as a vulnerable plaque (Figure $3 \mathrm{~A}$ ).

As the plaque was characterized as unstable based on the OCT aspect and was highly symptomatic at the same time, the lesion was treated by implanting a new, $3 \times 15 \mathrm{~mm}$ drug-eluting stent, preceded by balloon dilatation. Control OCT following stent placement showed good results, with compression of the plaque against the vessel wall, without luminal protrusion or edge dissections (Figure 3B).

At this stage, all therapeutic decisions were guided through OCT adjunctive imaging, leading to an optimal final outcome, with TIMI III flow in the entire coronary tree.

\section{DISCUSSION}

Acute coronary syndromes represent a major cause of cardiovascular morbidity and mortality, due to the significant reduction of myocardial perfusion, caused by the alteration in blood supply. ${ }^{6}$ The vast majority of ACSs result from the progression of an atherosclerotic process, a chronic condition that leads to plaque formation. ${ }^{7}$ The gold standard for emergency diagnosis in all ACSs is represented by coronary angiography, an invasive imaging technique that provides important information about the location and severity of the lesions, at the same time offering the possibility to perform the therapeutic intervention consisting in stent implantation immediately.

Besides coronary angiography, novel intracoronary imaging methods are able to provide a more accurate visualization of the lesion. OCT and intravascular ultrasound (IVUS) are two of the most widely used invasive imaging techniques, being useful in characterizing the vulnerability degree of coronary plaques, especially in patients who are considered to be at high risk of cardiovascular events. ${ }^{8}$ As the patients continue to be exposed to the risk of cardiovascular events due to stent thrombosis, stent restenosis, or the development of new stenoses in the coronary tree even after treating the acute condition, the careful follow-up of all patients with a history of stent implantation is of extreme importance. ${ }^{6}$ These new events can occur especially due to the dynamic nature of atheromatous plaque progression that can develop even faster after a stenting procedure.

OCT is an invasive imaging method that allows the interventionist to analyze the micromorphology of coronary arteries and the characteristics of restenotic tissue within the implanted stent. ${ }^{6,9,10}$ At the same time, OCT presents a high resolution and numerous advantages for the study of coronary stents such as the possibility of quantifying the stent diameter and area, or detecting coronary artery dissections or stent malposition. Also, OCT is the first-choice technique to measure the thickness of the fibrous cap (a significant predictor of coronary plaque vulnerability), and to assess other characteristics of plaque vulnerability such as macrophage infiltration, lipid accretion, intracoronary thrombus, or neointimal rupture. Compared to IVUS, OCT can also recognize calcium deposited in the coronary artery wall. However, its main impediment is poor axial penetration, which precludes a precise estimation of the vascular remodeling process. ${ }^{1,9,11}$

Furthermore, OCT is frequently used as a guide during coronary angiography and in the assessment of vascular response following a percutaneous coronary implantation. The association of OCT to standard angiography can help to detect the vulnerable plaques, providing vital information about the therapeutic strategy to follow, balloon selection, or stent dimensions. In bifurcation lesions, the reconstruction of OCT data into 3D images can determine the location of the main artery and the position of the side artery, guiding the wire progression across a side branch over the stent insertion in the main artery. ${ }^{10,11}$ In addition, OCT can appreciate several complications that can occur in the poststenting period, such as incomplete stent strut coverage, in-stent restenosis, stent thrombosis, or neoatherosclerosis, providing relevant insights into the process of progression or endothelial healing over the stented area, incomplete stent apposition or stent strut coverage..$^{11,12}$

However, ОCT has some limitations in the assessment of coronary plaque vulnerability. The major limitation, which results from the limited penetration, is the inability to recognize the necrotic cores of the vulnerable coronary plaque. Moreover, OCT may require supplementary contrast use due to the fact that blood flow may influence OCT images. ${ }^{11}$

In our case, OCT was extremely useful for differentiating a possible intra-stent thrombosis or restenosis from a vulnerable plaque, as a cause for angina symptoms. The coronary angiography aspect remained unchanged 
from the previous examination and showed no severe lesions, therefore the etiology of the new-onset angina remained unclear. OCT was able to identify the presence of a vulnerable plaque inside the coronary tree, which was responsible for the symptomatology, despite being hemodynamically non-significant. The entire revascularization procedure was guided by OCT, which was also useful for the evaluation of the previously implanted stents and their endothelial coverage, as well as the complete apposition of the newly implanted stent. This intracoronary imaging technique revealed permeable stents, without any signs of neointimal hyperplasia or neoatherosclerotic plaque formation inside the previously implanted stents, at the same time being able to identify the target lesion. The vulnerable plaque identified by OCT, with a large lipid pool and a thin fibrous cap, was the lesion responsible for the new onset of angina symptoms.

The case reported here represented a complex condition of a patient with severe recurrent angina pectoris and multiple cardiovascular comorbidities (type 2 diabetes mellitus and stage III chronic kidney disease). The particularity of this case consists in the fact that the patient presented at seven years after the implantation of a coronary stent with the same symptomatology, and the complex coronary artery imaging techniques demonstrated the progression of the disease with the development of multiple serial new stenoses, which required the implantation of new coronary stents. Moreover, after 1 year, the patient returned with severe chest pain caused by the development of a new vulnerable plaque. In this case, we faced an accelerated and progressive atherosclerotic process, probably augmented by the significant comorbidities.

\section{CONCLUSION}

This case accentuates the importance of the new intracoronary imaging techniques in monitoring the evolution of patients with implanted coronary stents. Cardiovascular comorbidities may have a direct influence on the atherosclerotic process, leading to the occurrence of new events, years after the acute episode. Invasive imaging techniques are currently considered extremely useful in achieving a personalized therapy in patients with coronary artery disease and high cardiovascular risk. One of the most important imaging techniques of this kind is OCT, which can provide relevant information on plaque vulnerability and intra-stent plaque formation.

\section{CONFLICT OF INTEREST}

Nothing to declare.

\section{REFERENCES}

1. Thom T, Haase N, Rosamond W, et al. Heart disease and stroke statistics - 2006 update: a report from the American Heart Association Statistics Committee and Stroke Statistics Subcommittee. Circulation 2006;113:e85-e151. doi: 10.1161/ CIRCULATIONAHA.105.171600.

2. Mackay J, Mensah GA. The Atlas of Heart Disease and Stroke. Available at: http://www.who.int/cardiovascular_diseases/ resources/atlas/en/

3. Benedek T, Mester A, Benedek A, Rat N, Opincariu D, Chitu M. Assessment of Coronary Plaque Vulnerability in Acute Coronary Syndromes using Optical Coherence Tomography and Intravascular Ultrasound. A Systematic Review. Journal of Cardiovascular Emergencies. 2016;2:173-184. doi: 10.1515/ jce-2016-0028.

4. Nyulas T, Morariu M, Chitu M, et al. Positive Remodeling as a Biomarker of Plaque Vulnerability-at the Border between Invasive and Noninvasive Assessment. Journal of Interdisciplinary Medicine. 2017;2:27-30. doi: 10.1515/jim2017-0021.

5. Kiyoshi H, Kazuo K, Satoshi U. Clinical utility and significance of intravascular Ultrasound and Optical Coherence Tomography in guiding percutaneous coronary interventions. Circ J. 2015;79:24-33. doi: 10.1253/circj.CJ-14-1044.

6. Zhang YJ, Pang S, Chen XY, et al. Comparison of intravascular ultrasound guided versus angiography guided drug eluting stent implantation: a systematic review and meta-analysis. BMC Cardiovasc Disord. 2015;15:153. doi: 10.1186/s12872-0150144-8.

7. Leite WF, Ramires JA, Moreira LF, Strunz CM, Mangione JA. Correlation between C-Reactive Protein in Peripheral Vein and Coronary Sinus in Stable and Unstable Angina. Arq Bras Cardiol. 2015;104:202-208. doi: 10.5935/abc.20140188.

8. Benedek E, Stanescu A, Orzan M, Rat N, Kovacs I, Suciu Zs. Characteristics of Neoatherosclerosis Within Implanted Coronary Stents in Patients with Acute Coronary Syndromes. Journal of Cardiovascular Emergencies. 2016;2:19-26. doi: 10.1515/jce-2016-0004.

9. MaT, Yu M, LiJ, etal.Multi-Frequency IntravascularUltrasound (IVUS) Imaging. IEEE Trans Ultrason Ferroelectr Freq Control. 2015;62:97-107. doi: 10.1109/TUFFC.2014.006679.

10. Garcì-Garcìa HM, Gogas $\mathrm{BD}$, Serruys PW, Bruining N. IVUS-based imaging modalities for tissue characterization: similarities and differences. Int $\mathrm{J}$ Cardiovasc Imaging. 2011;27:215-224. doi: 10.1007/s10554-010-9789-7.

11. Zhang BC, Karanasos A, Regar E. OCT demonstrating neoatherosclerosis as part of the continuous process of coronary artery disease. Herz. 2015;40:845-854. doi: 10.1007/ s00059-015-4343-y.

12. Mehanna EA, Attizzani GF, Kyono H, Hake M, Bezerra HG. Assessment of coronary stent by optical coherence tomography, methodology and definitions. Int J Cardiovasc Imaging. 2011;27:259-269. doi: 10.1007/s10554-010-9793-y. 